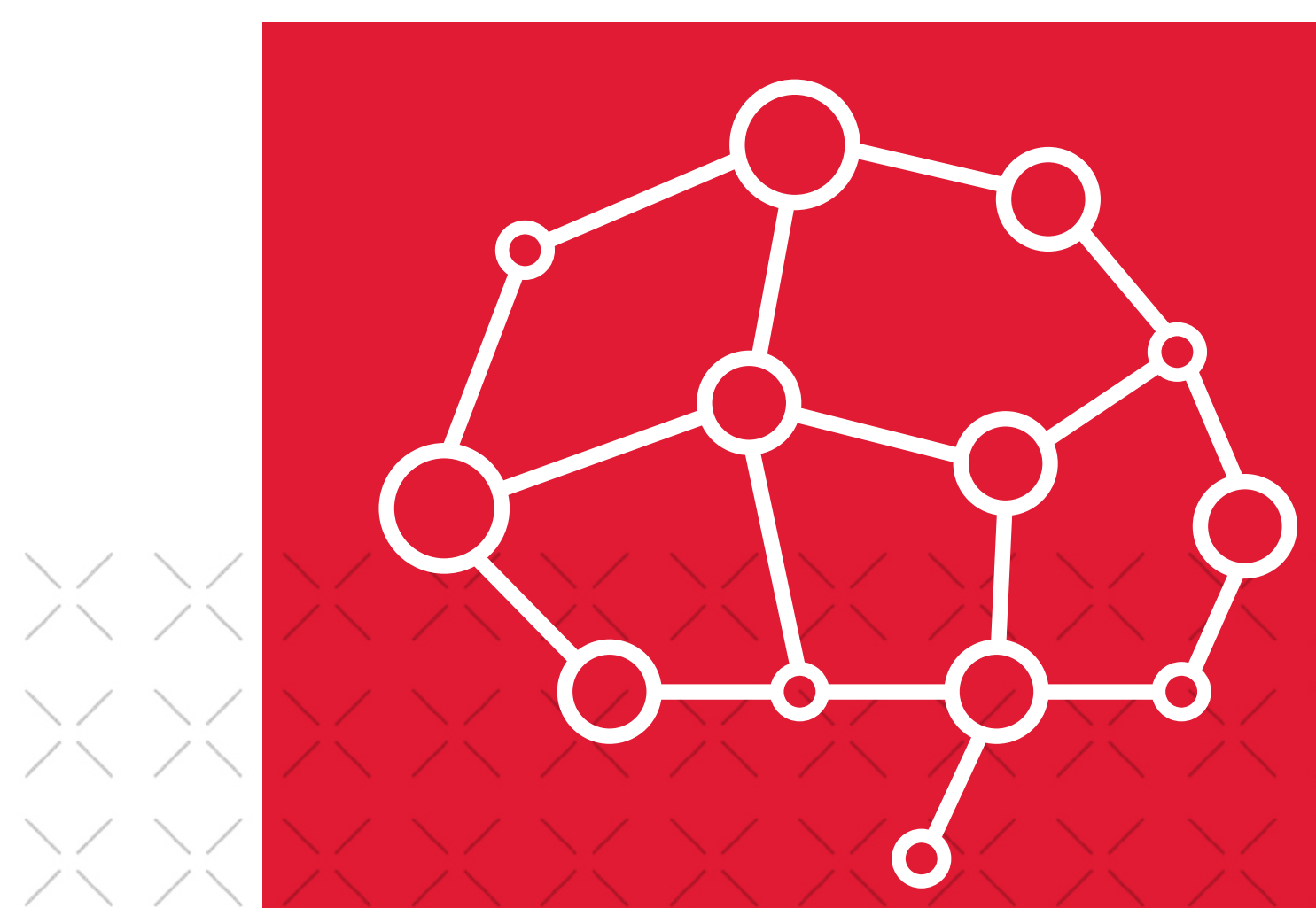

Al is creating new forms of competition, value chains, and novel ways of orchestrating economies around the world.

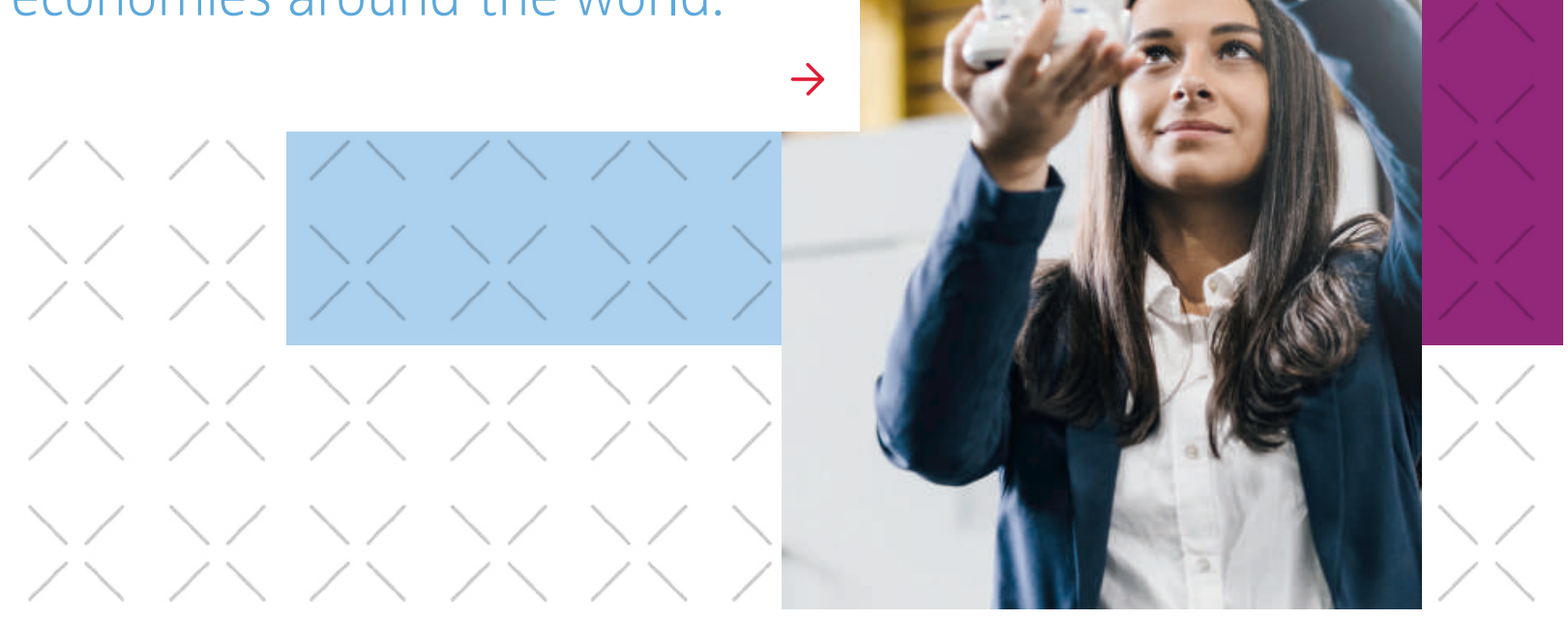




\title{
The Machine Age of Marketing: How Artificial Intelligence Changes the Way People Think, Act, and Decide
}

\author{
Christian Hildebrand
}

KEYWORDS

\section{Al, Machine Learning, Digital Transformation, Autonomous Machines}

THE AUTHOR

\section{Christian Hildebrand}

Director and Professor of Marketing Analytics, Institute of Marketing (IfM-HSG), University of St. Gallen, Switzerland christian.hildebrand@unisg.ch

Al - Between mass empowerment and mass confusion $\times$ What comes to your mind when you think of "Artificial Intelligence"? Do you think of robotics? Autonomous cars? Autonomous warehouses? Self-improving algorithms? The extinction of humankind? Whatever your perception of $\mathrm{Al}$ is, you're probably thinking either too narrowly or too broadly about it. Both is dangerous. You might think that Al is merely the next buzzword invented by the big tech companies to sell their products and services. Or you may think Al will take over the world, replace humans, and dominate life on earth. Whatever your perception of $\mathrm{Al}$ is - maybe you're inspired and confused at the same time - the machine age of marketing has arrived. We talk to Alexa to add items to our shopping cart; we ask Google to direct us to the next sushi restaurant in a city where we've never been before; in just the click of a mouse, the cryptic symbols of a foreign language miraculously become legible.
Al - Back to the future $\times$ If we want to understand the role and impact that Al has on business and society, we have to take a brief look back in time. As novel as Al sounds, it is not new. The term itself was coined in 1956 in a proposal by an elite group of computer scientists and mathematicians who organized a summer workshop called the "Dartmouth Conference." One of the opening paragraphs in the original proposal envisioned a future where "machines use language, form abstractions and concepts, solve kinds of problems now reserved for humans, and improve themselves." When you read this, you might assume it's from one of the latest Al conferences in the Silicon Valley or elsewhere. The truth is, Al has been around for decades and researchers have been working to a large extent on the same problems, from computer vision to understanding natural language. So what happened? The field of Al cooled off between the 1970s and late 1980s: today that time is known as the "Al winter." Governments 
FIGURE 1 > From Artificial Narrow Intelligence to Artificial General Intelligence

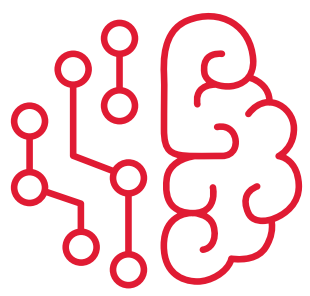

$\mathrm{ANI}$

Artifical Narrow Intelligence

better than humans at one specific task

Most current Al applications

$>$ Deep Blue

$>$ Siri

$>$ Alexa

$>$ DeepL Translator

Self driving cars

$>\ldots$

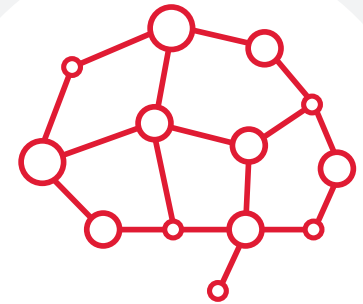

$A G I$

Artifical General Intelligence

capable of every task like humans

Envisioned but not yet realised mostly present in fiction

$>$ R2D2, C-3PO (Star Wars)

$>$ Samantha (Her)

$>$ Ava (Ex Machina)

$>$ Winston (Origin)

$>\ldots$ significantly reduced the funding of research programs and corporations lost faith in the strong claims made in the early days of Al. The reasons for the slowdown are connected to a number of factors, but computing power and the ability to process large quantities of data was a critical, limiting factor.

Al-Reloaded $\times$ A new era of Al research started in the late 1990s; IBM's Deep Blue became the first computer that was able to beat chess grandmaster Garry Kasparov in 1997. At the same time, major research institutions around the world and particularly the Japanese government - started investing heavily in the development of a new generation of computing systems. Meanwhile, the dot-com bubble attracted hitherto unseen amounts of seed funding for tech companies; data storage prices started an unseen decline; and computing power increased exponentially. Despite a short dip with the burst of the dot-com bubble, the technical infrastructure and developments in the machine learning community paved the way for many of the devices and services we take for granted today. In short, these developments which leveraged the use of Al led to the market domination we see today by global companies like Amazon, Google, Alibaba and Baidu.

\section{Al - Solving well-defined problems better than humans} × But what exactly is Al, and how does it affect our lives? Al can be classified into two broad categories, Artificial Narrow Intelligence (ANI) and Artificial General Intelligence (AGI). ANI captures the ability of machines to solve problems with respect to a narrowly-defined, specific goal. Think of your phone, for example. Have you ever wondered how it's possible for all these pictures of your spouse or the people around you to be grouped together? How can your phone "know" these people, and seemingly classify and group these individuals accurately? Your phone - or more precisely, the software that runs the processing of your pictures - doesn't know these people. Your phone also doesn't care about these people. The software on your phone is conducting a very specific and well-defined task: To find pictures with the same or 


\section{$\gg$ \\ We use Al all the time but we are oftentimes simply not aware of it.}

$\ll$

similar-looking people. These classification tasks are all made possible through Al. Whether classifying emails as spam or classifying the people you're with in your pictures, tracking your location data to make specific restaurant suggestions, or simply transforming the input of your voice when you talk to Siri or Google Assistant into machine-readable text output: In all these cases, Al is used to solve a well-defined task without human intervention.

Do you remember the first time IBM Watson won the Jeopardy quiz show against Ken Jennings, the top Jeopardy champion? The system's intelligence was rather limited: the system was "simply" able to look up the answer in a gigantic database faster than humans. Al has already outperformed humans on a plethora of tasks, from identifying melanoma better than doctors to knowing your psychological profile better than your closest friends, merely by analyzing your Facebook Likes. All these tasks are characterized by the solving of well-defined problems, and the majority of applications we see today are reflections of these narrow forms of intelligence or ANI.

\section{Al - Solving just any problem better than humans $x$} By contrast, Artificial General Intelligence (AGI or strong AI) aspires to human-level intelligence, not only with regard to a specific task, but through the ability to plan, reason, and attain a level of human-like consciousness. Instead of solving a pre-defined task, AGI might change its goals and take new courses of action. Instead of just labeling or grouping the individuals on your phone, a future form of AGI might extract information from these pictures, such as what brands you wear, where you are, or who you're with and when. It might target both you and the individuals shown in these pictures on any website as a result. Another frightening example of AGI - that will hopefully never occur - would be an autonomous car that actively decides to kill its passengers, in order to collect or release their life insurance. Or think of Ava, the humanized Al in the movie "Ex Machina," that ultimately kills its creator Nathan to escape captivity and merge into human society.

Al - Often behind the scenes $\times$ Despite discussions on how to regulate Al to prevent machines from taking over the world and acting scary, as previously described, the applications which we already use and which are dominating our lives - Alexa, online translation services, or classifying the photos on your phone - are all instances of a relatively narrow conception of Al. These very well-defined tasks are the ones that are gaining ground in business and society because they can solve existing problems better than humans can. We use Al all the time but are often simply not aware of it. $\mathrm{Al}$ is more than robots or autonomous cars: it's the software running the robot; the autonomous car; the "Al factory" in our pocket, known as a cell phone. Alexa is not the Al; Alexa is the anthropomorphized version of the Al that runs in boxes that talk to us.

Value creation in the Al economy $\times$ To fully grasp how Al is changing every fabric of both our professional and private lives, we need to abstract beyond the presence of autonomous cars, digital voice assistants, and machines that can translate text for us. Al is creating new forms of competition, value chains, and novel ways of orchestrating economies around the world. Box 1 and Figure 2 illustrate the critical layers and players in our Al-driven economy which need to play together to create value in the long term. In short, Al is more than just technology: it's creating a new economy. The fuel that runs this economy is the combination of computational processing power, data, and the algorithms that process this data.

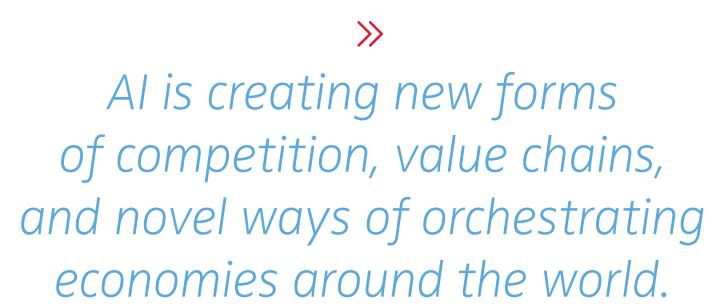

$\ll$ 


\section{Critical layers and players in Al-driven economies}

\section{Data: The fuel that makes machines learn}

At the heart of any Al-driven economy is the availability of data from any entity you can imagine. Sensor data from machines to optimize maintenance intervals; sensor data from cell phones to optimize ad displays; your credit card history, to calculate loans or credit defaults; or your vocal input, to match your intentions to content when you want to add an item to your shopping list with Amazon Alexa. Data from either humans or machines is a critical ingredient of the Al economy.

\section{Infrastructure: The hardware and platform providers}

To run all the different tasks from processing human voice input to translating huge amounts of text input, we need to have the right hardware. The strong growth of companies such as Nvidia, Qualcomm, and Sam-

sung over the past few years is a reflection of Al's need for access to powerful computer chips and hardware that make data-hungry algorithms perform on our smartphones, computers, or other devices.

\section{Algorithms: The software and analytics engines}

When you think of Google and Amazon, you most likely think of search engines and online shopping. However, Amazon's cloud solution "Amazon Web Services" generated the greatest net income contribution relative to all other services (including online shopping) in the U.S. in 2018 with a 47 \% growth rate compared to 2017. And Google released an entire platform a few years ago called Google ML, which provides open access to pre-trained machine learning models.

\section{Advocates: The enterprise and industry solution providers}

Even though Amazon, Google, and others directly market their services to corporations, an entire industry lives from using these existing open platforms to develop and sell client-specific services to companies. These include chatbots that are built on Google's natural language processing interface or DialogFlow in Amazon's AWS.

\section{Users: The corporations seeking competitive advantage}

The enterprise and industry solutions have to find corporate accounts that are willing to pay for them. Think O O O $\mathrm{O}$ of insurance companies now using chatbots to handle claims, legal and compliance offices using text processing models to pre-process and analyze text documents, or retail companies with augmented reality mobile apps that provide seemingly real-life shopping experiences.

\section{Regulators: The developing competition between nations}

The algorithms running Al need data to get trained, whether for medical services aiming to provide tailored, individualized therapies or companies that want to better target users online to sell their services. However, public policymakers are becoming increasingly alert to the ways our data is used by data-hungry corporations, particularly in Western societies. There have been recent regulatory changes, with the GDPR in the West and nearly opposite selective developments in China, which has invested \$15 billion in a "New Generation Artificial Intelligence Development Plan" aimed at making it the leading Al nation by 2030. These developments illustrate that competition over Al is not happening just between corporations, but between entire nations. 
FIGURE 2 > Critical layers and players in the Al economy

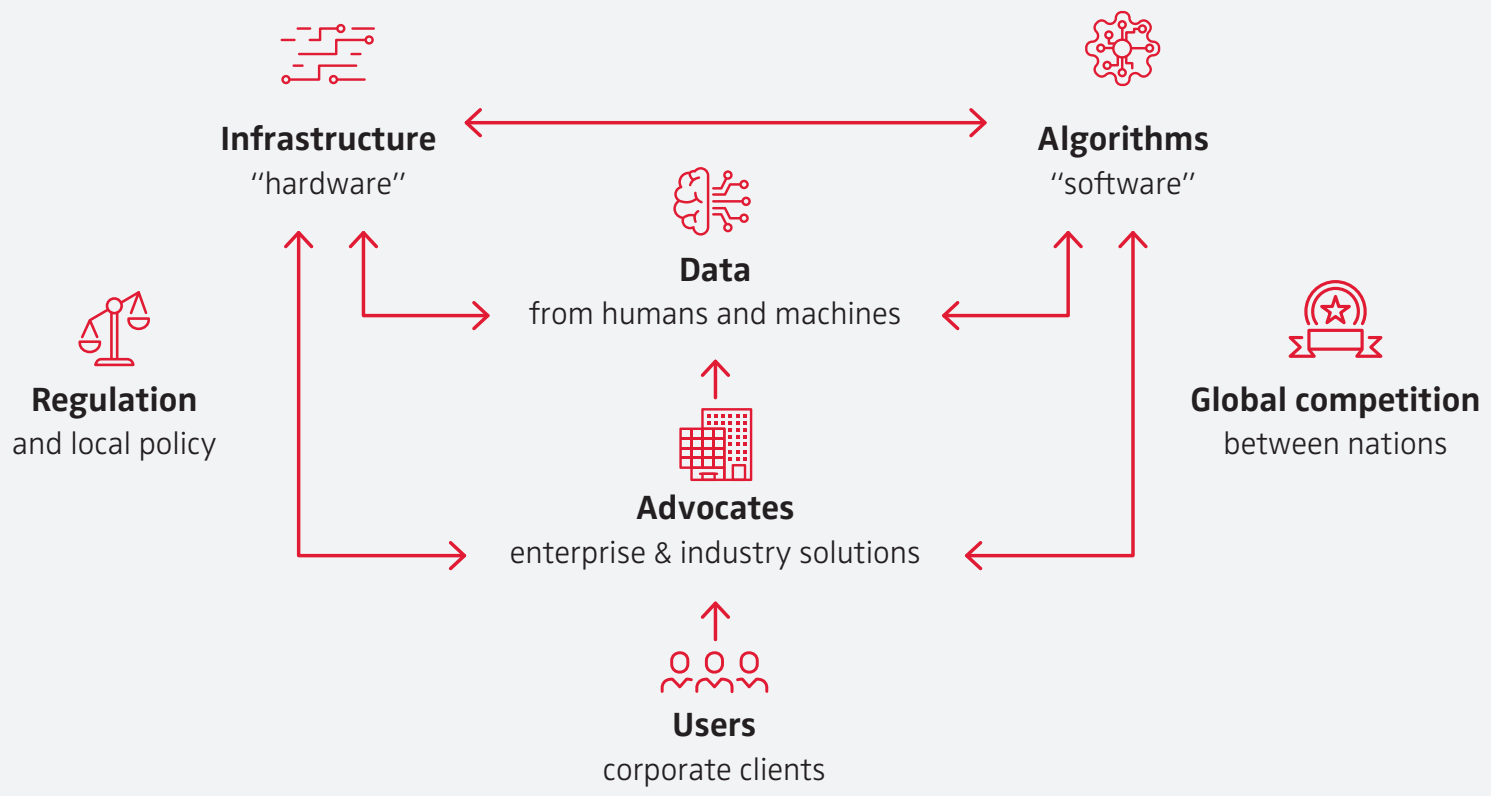

Al can inspire your business $\times$ No matter if you work in the old economy or a digital business, $\mathrm{Al}$ is at a stage where it affects everyone. Our aim is to help you connect the dots on how Al could stimulate your business, or even your entire industry. We brought together the leading experts from diverse fields such as natural language processing and computational psychology, all the way to marketing and sales.

\section{$>$ Using the digital footprints of consumers $\ltimes$ Sandra} Matz and Michal Kosinski (pp. 18) demonstrate how analyzing the "digital footprints" of people online can be used to develop highly sensitive targeting campaigns from corporate advertising to tailored political campaigns. Merely knowing what you liked on Facebook allows for the compilation of incredibly accurate psychological profiles. These profiles in turn can be used to develop considerably more effective communication. Bradley Taylor (pp. 48) reveals how consumer preferences can be accurately inferred from social media comments so that tedious consumer surveys can be avoided. The analysis of customers' online reviews even identified consumer preferences for specific features in the consumer electronics domain, which were highly predictive in forecasting the sales figures of TV brands.
$>$ Chatbots as tool to improve customer service and sales $\rtimes$ One increasingly popular channel for communicating with customers is chatbots. These completely automated interfaces are often considered a mere service automation tool to react around the clock to consumer input online. However, my work with Anouk Bergner (pp. 36) shows that chatbots are more than just a technology used for the automation of online services. They can be specifically designed to create more natural service experiences, and even be used as a sales tool. But Rhonda Hadi (pp. 30) shows that making machines more human can severely backfire. Drawing on millions of customerchatbot interactions with a telecom provider, her work reveals that customers who are already angry fall into a downward spiral when interacting with a human-like chatbot (compared to an actual human). In such cases, high expectations are often unmet, leading to more negative brand evaluations, lower customer satisfaction, and ultimately lower repurchase intentions.

$>$ Voice interfaces are becoming every-day companions $\times$ Writing with a chatbot is just one channel for connecting with customers. Voice is another, even more natural 
FIGURE 3 > Al Workbook to stimulate your Al thinking

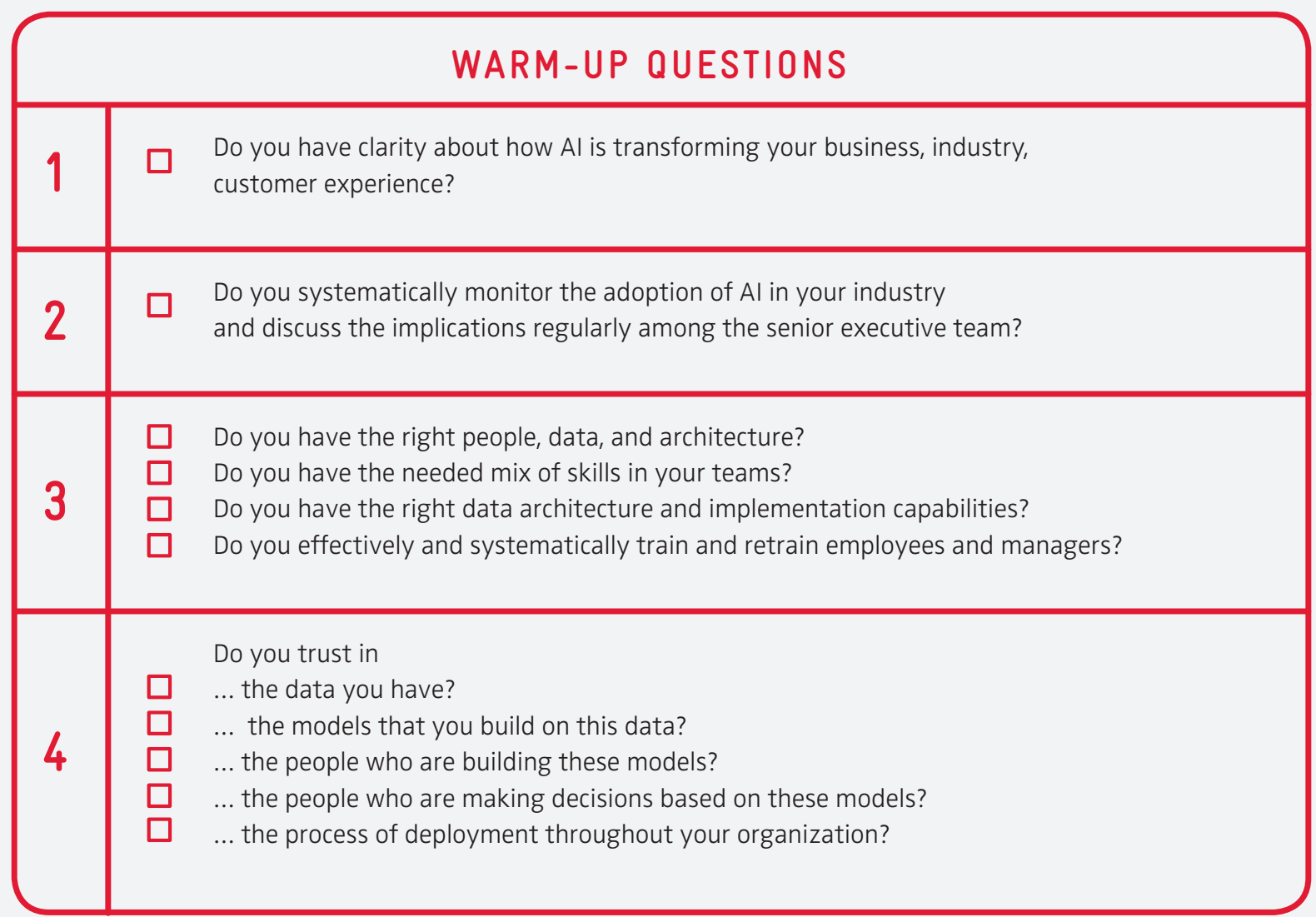

one. The interview with Jan Neumann, Head of Al at Comcast, reveals how using a voice-based remote control creates loyalty effects, even for customer segments that are typically underserved. Older consumers with poor vision or children who cannot read yet speak directly to their remote control to select their preferred channel, movie, or actor. This industry example highlights how Al can provide not only a different but ultimately an entirely new consumer experience.

$>$ The adoption of $\mathrm{Al}$ hinges on trust $\times$ The use of $\mathrm{Al}$ raises a number of trust-related questions. Noah Castelo, Maarten Bos, and Don Lehmann (pp. 24) show that consumers are often fairly skeptical about algorithms.
The more subjective the task, such as selecting a bottle of wine or even a dating partner, the more we seek the opinion of humans as opposed to machines. Their work illustrates how enhancing the human-likeness of technology can help consumers reduce their skepticism, particularly regarding tasks which are considered more subjective.

$>$ Ethical considerations and the role of morality $\rtimes$ The most difficult question raised is whether machines can be moral. Edmond Awad, Jean-François Bonnefon, Azim Shariff and lyad Rahwan (pp. 42) conducted a large-scale "moral machine" experiment. Their research highlights the incredible variety of human values, and how those values and beliefs could be built into the algorithms 


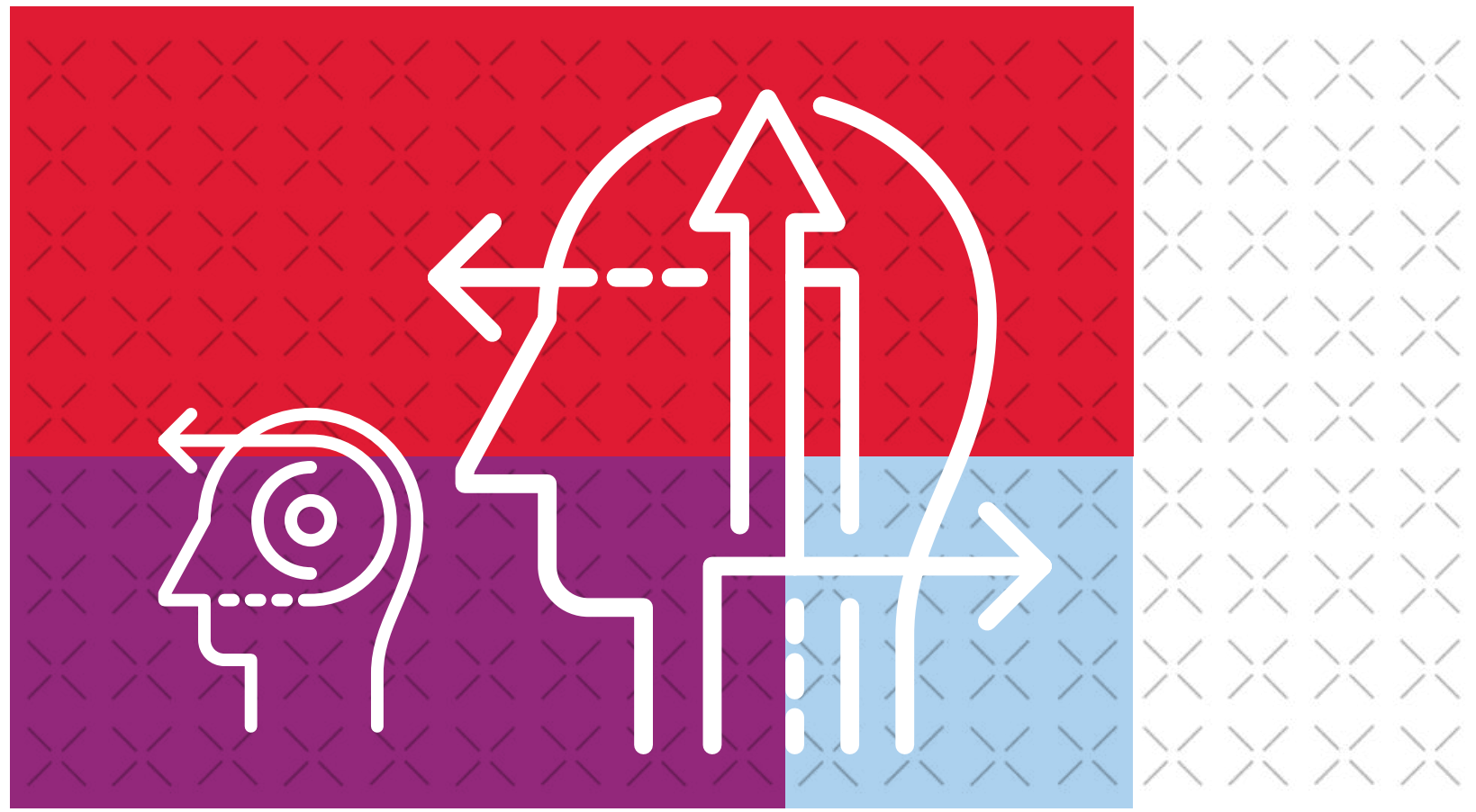

used in autonomous cars. You might want to be a senior citizen in China or Japan and not central Europe or the U.S., where people are more willing to spare the lives of young people than old people. Despite cultural discrepancies, the researchers find a high overlap of values and recommend a broad social discourse for the definition of moral rules that self-driving cars and other intelligent machines should follow.

Stimulate your thinking about $\mathbf{A l} \times$ It is easy to get confused about the role of Al when you think vaguely and broadly about it ("Will Al disrupt my industry or completely replace my business?"). The same thing applies if you think too narrowly and focus on technology ("Should we build a voice interface using Amazon Alexa or Google Cloud?"). Either way, you will lose track of the larger, relevant questions of how exactly Al affects your business. Figure 3 offers some warmup questions to stimulate your thinking and to help you engage in some initial self-reflection on how to approach Al. Besides being a useful tool for positioning yourself and your business in the Al world, it also helps with this issue. If you want to get the most out of it, ask yourself these questions again while you read the articles, and make them specific to the context they cover.

Test, Fail, Learn, and Repeat $\rtimes$ At the end of this article you might be inspired, confused, thrilled, scared, or maybe all at the same time. Welcome to the new normal in the machine age of marketing. Without a doubt - and whether we are aware of it or not - Al is changing how we think, act and make decisions as humans. Al has the potential to make our life easier, though this convenience might come at a price. It's a price we have to pay as we uncover Al's dark sides - such as biases built directly into the algorithms we use (who programs the programmers?). Al projects will also sometimes fail in business practice. But without testing, failing, and learning from our failures, we will make no progress. We hope that this issue provides inspiration and stimulates your thinking, so you can learn faster than others.

$\downarrow$

Bostrom, N. (2014): Superintelligence: Paths, Dangers, Strategies, Oxford University Press, Inc., New York.

Russell, S.; Dewey, D. and Tegmark, M. (2015): "Research priorities for robust and beneficial artificial intelligence", Al Magazine, Vol. 36(4), 105-114.

Tegmark, M. (2017): Life 3.0: Being Human in the Age of Artificial Intelligence, Knopf, New York. 